\title{
The Influence of Mobile Learning on the Optimization of Teaching Mode in Higher Education
}

\author{
Xining Zhang (10) \\ College of Education Science, Weinan Normal University, Weinan, Shaanxi, China \\ Correspondence should be addressed to Xining Zhang; 201904020314@stu.zjsru.edu.cn
}

Received 12 November 2021; Accepted 25 November 2021; Published 27 January 2022

Academic Editor: Narasimhan Venkateswaran

Copyright (c) 2022 Xining Zhang. This is an open access article distributed under the Creative Commons Attribution License, which permits unrestricted use, distribution, and reproduction in any medium, provided the original work is properly cited.

\begin{abstract}
The purpose of the study is to optimize the teaching mode of higher education. The teaching mode of colleges and the learning situation of college students are studied based on mobile learning. Artificial neural network (ANN) algorithm is implemented to train and test the student responses. The classification results show that compared with the Traditional Teaching Mode (TTM), the teaching mode of mobile learning can significantly improve students' learning effectiveness, skill mastery, and learning enthusiasm. Compared with the TTM, the teaching mode of mobile learning can help college students get a better learning experience. The number of students participated or attended the classes through mobile applications is higher when compared with the other traditional methods.
\end{abstract}

\section{Introduction}

China's higher education gradually begins to have a complete system after the reform and opening up. However, the world has gradually entered the information age with the continuous popularization of Internet technology and computers worldwide. Besides, the industrial impact brought by the technological revolution will quickly spread to any country in the world after the development of economic globalization. More and more scholars begin to realize the importance of scientific as well as technological innovation. Besides, accelerating the process of modernization as well as promoting the construction of digitization and informatization has become the main guiding direction of all walks of life [1]. The field of education also faces great challenges. The development of education will seriously affect a country's future as well as destiny. Continuous reform and innovation of education is the inevitable requirement of the development of the times. The TTM is no longer suitable for students growing up in the new era environment, so the hybrid teaching mode begins to gradually come into people's vision, and the mobile learning mode with Internet technology as the main core has become a hot field in the current education field [2]. Nowadays, mobile learning has become the general trend of higher education teaching model reform, and it is also one of the main forms to promote the modernization and informatization of higher education. It is becoming increasingly common for people to use mobile devices for personal communication, which is speeding the shift away from desktop web-based applications. The high-speed central processor unit of today's mobile devices provides significant computational capabilities (CPU). Wearable gadgets such as smart watches and wrist-worn devices as well as mobile devices such as smartphones and portable PCs have become ubiquitous in mobile learning [3]. A wide range of multimedia service applications are supported by these devices, which have user-friendly human-computer interfaces and modes of operation. Different types of network resources can be accessed by the devices via a variety of network connection methods. In terms of using these devices as learning tools, they have been used in a range of settings, including formal and informal learning situations.

The purpose of educational technology is to help students learn more effectively and efficiently by utilizing the right technology. The use of Learning Management Systems (LMSs), Intelligent Tutoring Systems (ITSs), smart boards, social media, and blogs in higher education has lately been implemented. The author indicates that educational 
platforms should be established in order to help students develop their educational literacy as well as adapt education to the growing importance of modern technology [4]. Enhanced educational environments are better able to give students with the teaching and learning capabilities they require at the appropriate times. Educational technology is critical in preparing students for the challenges of today's and tomorrow's workforce. Implementing these technologies requires a significant amount of labour, planning, and financial resources [5].

As one of the most cutting-edge innovations in educational technology, m-learning provides students and teachers alike with an abundance of options. A number of institutions throughout the world have used $m$-learning, a method of delivering learning regardless of place or time. Using tablet computers and PDAs, Abilene Christian University's students and faculty members have been using Apple devices as part of the Mobile Learning Initiative (MLI). To teach French grammar and vocabulary to undergraduate students, Princess Nora University has used mobile phones [6]. King Saud University's educational programmes have taken advantage of its apps by using mobile devices. There is a strong case to be made for the use of mobile learning in higher education [7]. M-learning will have the ability to adapt many different educational methods soon.

When it comes to personal communication, people increasingly choose to utilize their mobile devices rather than their computers. The high-speed central processor unit of today's mobile devices provides significant computational capabilities (CPU). Wrist-worn and other mobile gadgets such as mobile phones and portable computers have become prevalent in mobile learning [8]. Pen tablet computers and laptop computers are also commonplace in mobile learning. These devices, which implement convenient multimedia service applications, provide user-friendly human-computer interfaces and operation modes. The devices can also gain access to a wide range of network resources by using a variety of network connection strategies. From small-scale, short-term trials to large-scale deployments of these devices, they have been employed in both formal and informal learning situations among all the excitement around mobile learning; its notion remains undefined and undefined [9]. Mobile learning solutions like SMS and Facebook applications can help higher education institutions deal with rapid technological development, competition, and globalization, as well as globalization in the workplace. In order to help students in higher education learn more effectively, this article discusses how SMS and Facebook mobile learning can be combined with traditional classroom instruction. The majority of college students use SMS and Facebook as their primary methods of communication. To reach as many students as possible, this is in line with one of the primary goals of mobile learning [10]. In addition, SMS and Facebook are both popular and viewed as useful in meeting the needs of students, making them a valuable resource.

Effective planning and implementation are essential if technological developments are to have a significant impact on education. When it comes to "mobile education," there are many different ways to look at the term. When discuss- ing mobile learning, some argue that it is an amalgamation of e-learning and mobile computer technology [11]. "Unique e-learning, confined by a number of special traits and the capabilities and bandwidth of devices, and other aspects of the network technologies that are being deployed," according to a variety of experts. Mobile devices such as PDAs and digital cell phones can be used to deliver e-learning, but some argue that it is just e-learning supplied via wireless transmission and mobile devices [12]. Although mobile learning can be described as the use of portable electronic aids, such as smart phones or PDAs, in academic circles, the phrase is typically used to refer just to the use of these devices, rather than the use of a computer or classroom setting.

Despite the fact that integrating technology in higher education has a great deal of potential, it can be challenging to think about how to integrate technology into the design of student learning [13]. It is unknown why some students are actively involved and have a better educational experience when using technology-supported learning, while others are not, and why this is the case. What factors play a role in the successful implementation of technology in education? In our opinion, there are several critical issues to consider when considering how teachers in higher education use technology in the classroom, and we feel they should be made aware of these considerations. Inextricably linked to the way university professors view technology is their understanding of what constitutes a successful application of that technology [14]. While colleges and universities are subject to a range of external influences on how they conduct business, it is critical to understand the elements that influence the use of technology for teaching and learning, as well as how academic staff may be supported and improved in their efforts [15]. As a result of a lack of attention paid to pedagogical considerations, an excessive emphasis has been placed on technological manifestations (in other words, on the instructional tools that are used). Examples include questions such as how and why students and teachers can benefit from the usage of technology. Despite the fact that e-learning technologies and online learning environments have been widely accepted in universities in Westernized countries, the pedagogical issues associated with them have been given only secondary consideration. It is not unusual to be taken aback by the complexity of the puzzle. As a relatively new medium, the researcher sums up his idea that communication technologies exerted more impact on society through their qualities than through the material they delivered. Oversimplifying the social link between medium and message and society in general, his technologically deterministic vision is oversimplified. If you take a deterministic approach to education, then media has a greater impact on student achievement than the efforts of educators and curriculum designers combined [16]. While the medium and the message are often at odds, it is more often than not a combination of both. In contrast to these findings, other scholars have concentrated on the distinctive contributions to educational processes and results made possible by diverse media technologies. It is not only because of Internet connectivity and the World Wide Web that technological 


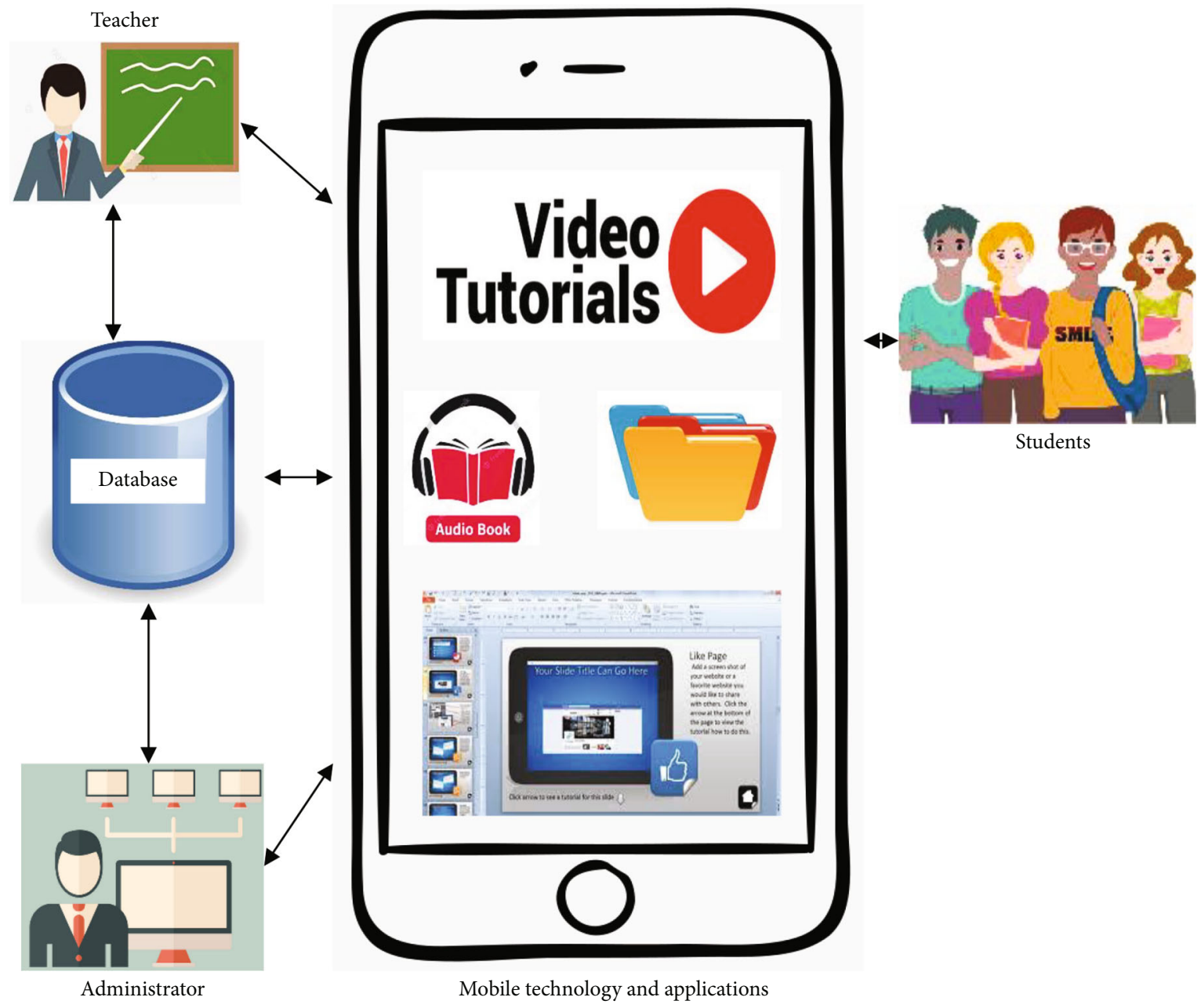

FIgURE 1: Architecture diagram of the proposed system.

advancements have been more readily available in educational settings, but it has also inspired the development of an increasingly wide spectrum of media technologies [17]. Students and professors alike are increasingly turning to technologically advanced mobile gadgets. Researchers are very interested in the effects of these technologies on today's teaching and learning environment. It is hard to overstate the influence that m-learning has had on education. Colleges around the world, including the United States, have begun using the new technology. This study focused on determining the influence of mobile learning in higher education.

\section{Materials and Methods}

The following are the attributes used for implementing the artificial neural network in the study.

(1) The number of epochs is 5 , the number of classes is 10 , and the batch size is 128

(2) Input video/audio measurement 2828

(3) Loading the data set's input audio/video
(4) Variable Exploration. $X$ represents the test data set $(15000,28,28,1)$, and $k$ represents the train data set $(90000,28,28,1)$

(5) Create and compile models

(6) Network training

\section{Proposed System}

Mobile Learning Technology has made it easy to study for the students of higher education (Figure 1). The teachers of the modern education system have utilized the development in information technology by accessing PowerPoint presentations, video tutorials, audio tutorials, and document circulation. Some teachers prefer to use the chalk and board in the live or online classroom session as the traditional way. When the teacher chooses video, audio, and document tutorials, the teacher can load the course materials in the database, supporting active intelligence. Teachers can use these modes of teaching, either online or offline, methods of education. The teaching and learning process can be of direct instruction, inquiry-based approach, or cooperative learning. Among these three types of processes, direct instruction 
is teacher-centric, whereas the other two methods are student-centric of teaching and learning. In direct instruction, the teacher will gain complete focus. Whereas in student-centric, the focus is shared among the student and the teachers. In the earlier case, the teacher will provide online lecturing of the course materials. The teacher may use one or more modes to make the student understand the concepts. However, in the later methods, students will share their knowledge with the supervision of the teacher. Latter methods will show improved performance in comparison with the earlier. However, some students may face difficulties in understanding or attending the classes when there is a power failure, unable to attend classes due to ill health, difficulty attending the course in small screen size, and so on. Another advantage of the cooperative learning method is the teacher will handle the class; then, the teacher will assess the students to think, discuss, and apply the concepts. All the course materials of the teacher will be made available in the database, and they can access the materials through the mobile application. Once the course schedule is uploaded, an intelligent system will be incorporated to auto-play the course materials as per the schedule.

The preceding algorithm explains the general steps involved in training and testing the image classification data set in ANN.

ANN considers as a dataset Ds and its containing $s$ objects $\left\{a_{1}, a_{2}, \cdots, a_{s 1}\right\} a_{i}(1 \leq i \leq s)$.

$F\left(R_{j}\right) \rightarrow$ defined as attribute is expressed in Equation (1).

$V \rightarrow$ variables

$P \rightarrow$ Probability of $R_{i}$ and $R_{j}$

$$
\begin{aligned}
F\left(R_{j}\right) & =-\sum_{v \in \text { domain }\left(R_{j}\right)} Y\left(R_{j}=h\right) \log Y\left(R_{j}=h\right), \\
L\left(R_{i i} R\right) & =F\left(R_{i}\right)+F\left(R_{j}\right)-F\left(R_{i} R\right), \\
1 & \leq i_{i} j \leq n(i \neq j),
\end{aligned}
$$

$n \rightarrow$ It represent as attributes as well as $n$ is objects of $\left\{a_{1}, a_{2}, \cdots, a_{n}\right\}$, where ai $\left(a_{i 1}, a_{i 2}, \cdots, a_{\text {in }}\right)$, differential of $a_{0}$ is represented in Equation (4).

$$
\begin{aligned}
\widehat{h}\left(a_{o}\right)= & \sum_{i=1}^{n} T_{x}\left(d_{i}\right)\left(\log x-\frac{x}{y} \log y\right)-a T_{a}(d) \\
& +a \sum_{i=1}^{n} F R\left(a_{o_{i} i}\right)
\end{aligned}
$$

where

$$
\begin{aligned}
& T\left(d_{i}\right)=2\left(1-\frac{1}{1+\exp \left(-F_{a}\left(d_{i}\right)\right)}\right), \\
& T_{a}(d)=\sum_{i=1}^{n} T_{a}\left(d_{i}\right) F_{a}\left(d_{i}\right) .
\end{aligned}
$$

The primary benefit is that $T_{a}\left(d_{i}\right)$ is a collection of data framework, assuming that performance management $s$ is genuine as in Equation (6). In Equations (4) and Equation (6), the most common lossy abilities compression framework used today, ANN of the two-dimensional in academic training/teaching handling is used.

$$
\begin{gathered}
F\left(a_{o \mathrm{i}}\right)=\left\{\begin{array}{ll}
0, & \text { if } n\left(a_{o_{\mathrm{i}} i}\right)=1 \\
\delta, & \&\left[n\left(a_{o_{\mathrm{i} i}}\right)\right] \text { iotherwise }
\end{array},\right. \\
\delta(a)=(a-1) \log (a-1)-\operatorname{alog}(a), \\
\widehat{h}\left(a_{o}\right)=\sum_{i=1}^{n}\left(\log x-\frac{x}{y} \log y\right)-x F_{x}(y)+x \sum_{i=1}^{n} F\left(a_{o_{i} i}\right) .
\end{gathered}
$$

In Equation (9), a function is calculated that ensures by having to add random qualities to a two coefficients. The greater the value of $x$, the greater the strength. Method for implantation and extraction

$$
\begin{aligned}
D s_{a} & =\frac{D s-D s_{\min }}{D s_{\max }-D s_{\min }}, \\
D s_{a} & =\frac{D s-D s_{\text {mean }}}{\sigma_{d}} .
\end{aligned}
$$

The technique mentioned above is that if Equation (10) does not really ignore the $D s_{a}$ audio/video, the correlations cannot be implemented to the desired link without accruing harms to a data contained in the specific.

$$
d_{\min }=\int\left(T^{x} \cdot F+R\right) \text {. }
$$

Essential research concluded that five classes were a reasonable trade-off for $d_{\text {min }}$ duration as in Equation (12): long enough to recognize gadget types but short enough to be entirely loaded up with some interesting multipacks from $d_{\text {min }}$. However, if $d_{\text {min }}$ does not contain sufficient outstanding packages to fill $d_{\text {min }}$, protecting with 0 qualities is used to determine the size is standouts.

$$
Y_{\text {copy }}=Y\left(d_{\text {min }}=1 \mid F\right)=\frac{\exp \left(T^{x} \cdot F+R\right)}{1+\exp \left(T^{x} \cdot F+R\right)} .
$$

A three $Y_{\text {class }}$ scheme is able to adapt but is also relevant for a growing number of device types. In Equation (13), training a single classifier for every device type is performed initially. Each classifier provides a paired option as to whether the input unique mark impression corresponds to the device category or otherwise.

$$
\log i t\left(Y_{\text {class }}\right)=\log \left(\frac{Y_{\text {class }}}{1-Y_{\text {class }}}\right)=T^{x} \cdot F+R .
$$

A few classifiers can recognize an Equation (14) obscure unique mark perception and thus coordinate some few device types. In such instances, $1 \log i t\left(Y_{\text {class }}\right)$ is used to cast the deciding vote between different matches by employing an alter separation based metric. While change separation can be used 
to identify device types on its own, it is more time consuming than order detachment of Equation (15).

$$
d_{\min }= \begin{cases}1, & \log i t\left(Y_{\text {class }}\right)>0.5 \\ 0, & \log i t\left(Y_{\text {class }}\right)<0.5\end{cases}
$$

The smaller the difference, the more accurately the learning resource's specialist knowledge points contest rand $a m_{j}$ $E_{i j}^{n}(r)$ the learner's information marks which is given in Equation (16).

$$
F_{i}^{n}(r)=\sum_{j \in L} \operatorname{rand} a m_{j} F_{i j}^{n}(D s)
$$

$(u, T ; R, \phi)$ the optimization technique of spending both with teaching programs represents the overall expenditure information between educational methods is calculated as in Equation (17).

$$
(u, T ; R, \phi)=|d|^{-0.6} \int_{-\infty}^{+\infty} d(\tau) h(\tau-r) e^{-j b \tau} d \tau .
$$

The principal function timeframe of education $L_{s}(u)$ objectives to clarify the difference in learning time required to complete teaching materials $F_{i} R_{i}(u)=Y^{R} R(u)$, and learning latency is given in Equation (18).

$$
L_{s}(u)=\sum_{i=1}^{n} F_{i} R_{i}(u)=Y^{R} R(u)
$$

The learner's total optimal control performance and also $\phi_{s, n}$ the learning path created more by comment thread function through recalibrating coefficient values, as demonstrated by Equation (19), which is a functional illustrative example of the personalized learning navigation optimization technique

$$
\Phi_{s, n}=\frac{\left\|R_{s, n}\right\|^{2}}{\delta^{2}} \operatorname{exps}\left(\frac{\left(R_{s, n} * F\right)}{3 \delta^{2}}\right) *\left[e^{i\left(R_{s, n} * Y\right)}-e^{-\left(\delta^{2} / 3\right)}\right] .
$$

Under the data quantity of each data record, it contains ten thousand data, the specified limit of higher education measurement, and decision-making distribution. The variance of evaluation as well as decision-making prediction error is level when $T$ is 1.7-1.9. The specified level of evaluation as well as decision-making diffusion can consistently keep the evaluation but also decision-making prediction error below $8 \%$. The Euclidian spacing of various classifications of higher education assets under data quantities of 200, 400, 600, and 800 specified levels of $11.29 \%, 14.52 \%, 20.97 \%$, and $24.19 \%$, respectively, of higher education resource evaluation, and decision-making diffusion is integrated within one class. The new classification can increase the efficiency as well as precision of data quantity 1000 data's specified level of $29.03 \%$ higher education resource evaluation but instead decision- making through data mining, thereby reducing error in higher learning resource evaluation or rather decision-making.

In general, modern higher education must meet the thinking mode and learning habits of college students in the new era and help improve students' initiative and enhance learning effect and learning experience; the reform of the teaching mode of higher education is imperative. Generally, the quality of a teaching model is mainly measured by students' learning effect and mastery of skills. Moreover, students' satisfaction and learning enthusiasm should be considered. Mobile learning can not only provide rich learning resources but also break through the constraints of TTM, break the limitations of time as well as space, and help students achieve a good experience of learning anytime and anywhere. Besides, the interactive classroom and interesting scenes of mobile learning can effectively promote students' learning interest as well as initiative. Compared with the boring traditional classroom teaching mode, mobile learning can better meet the inner demands of students. Moreover, for teachers, many functions of online learning can help to realize a simple and fast teaching process. The functions of punch in, roll call, and online Q\&A not only save classroom time but also help teachers quickly enter the teaching state. Meanwhile, the one-to-one teaching mode can also help teachers understand students' learning in time and modify the course progress based on students' mastery of knowledge. More importantly, many teaching materials and assignments are in the form of documents and PPT. Online learning can provide the function of one-click download, which saves the time to take notes in class and brings a lot of convenience to learning. Meanwhile, Internet technology also supports massive learning videos to be saved and downloaded. Students can watch some crucial content repeatedly without bothering the teacher to explain them for many times. It brings convenience to both teachers and students, enhances students' understanding and memory, greatly improves the learning effect, stimulates students' initiative and enthusiasm, realizes the maximum utilization of educational resources, and cultivates students' desire to explore knowledge and self-study ability.

\section{Results and Discussion}

In this study, the data has been evaluated by using artificial neural network. Figure 2 depicts the assessment but also decision accuracy of higher learning resource management under data analysis.

When applied to the learning system, the higher education evaluation time(s) and stance method based on data extraction will not only efficiently carry out intelligent parallel evaluation and outcome, but it will also make big data evaluation and selection among all educational methods into a device for user notification (Table 1).

The study determined why some people do not fill out the questionnaire; they can modify the study's data collection method to encourage reliable responses. Despite detailed planning and conscientious execution of solid models, researchers frequently end up with datasets for which they have no idea 


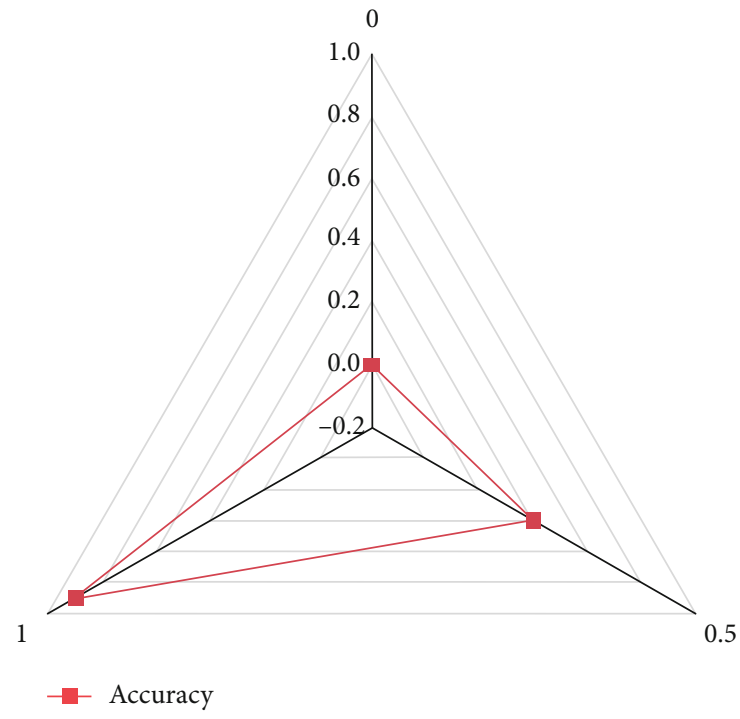

Figure 2: Performance analysis for data volume and evaluation time for higher education.

Table 1: Relationship between data volume and evaluation and higher education.

\begin{tabular}{lc}
\hline Data quantity (ten thousand) & Evaluation and decision time (S) \\
\hline 0.2 & 8 \\
0.4 & 11 \\
0.6 & 15 \\
0.8 & 17 \\
1 & 22 \\
\hline
\end{tabular}

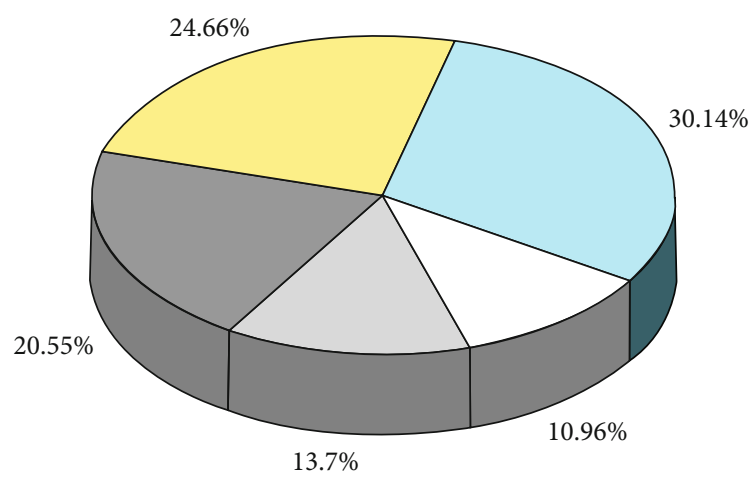

Performance analysis of online learning for higher educatin

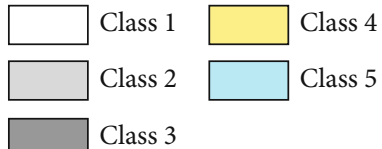

FIGURE 3: Performance analysis of online learning for higher education.

why such data are lacking, based on the data quantity evaluation and decision time to calculate.

The relationship between percentage and storage space of higher education evaluation and selection in the context
TABLe 2: Analyze the result data analysis of online learning for higher education.

\begin{tabular}{lc}
\hline The total number of evaluations and decisions & Accuracy $(\%)$ \\
\hline Class 1 & 10.96 \\
Class 2 & 13.7 \\
Class 3 & 20.55 \\
Class 4 & 24.66 \\
Class 5 & 30.14 \\
\hline
\end{tabular}

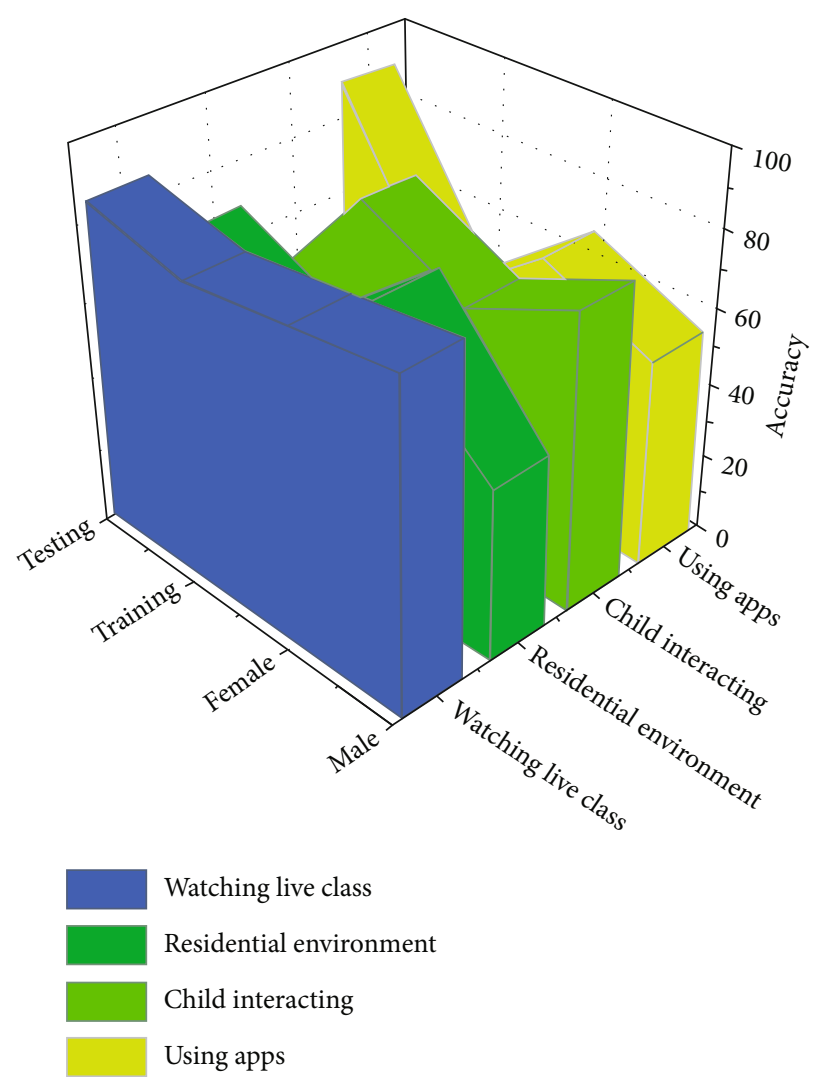

FIgURe 4: Performance analysis of mobile learning on the optimization of teaching for higher education.

of data extraction is represented in Figure 3. One will see that the increased number of scientific methodologies has little effect just on collection surface is covered by them, demonstrating that higher education evaluation and stance techniques under data analysis were also organized and feasible (Table 2).

The precision and certainty of an estimated statistic (e.g., mean, standard deviation, mean difference, and standard deviation difference) reflect a true value of the parameter, which is represented by, instead of standard errors, the mean value. A missing data instance reflects the clear and specific exclusion of a particular value for a specific variable within a particular case, introducing ambiguity into an approximate of statistical parameters used to calculate the accuracy. When executed on an education platform, a data exploration and production education evaluation and decision-making method will not only involve carrying out intelligent similar 
TABLE 3: Performance result analysis of mobile learning on the optimization of teaching for higher education.

\begin{tabular}{lccc}
\hline Parameters & Classification & Count & Percentage (\%) \\
\hline \multirow{2}{*}{ Watching live class } & Male & 345 & 85.65 \\
& Female & 573 & 81.53 \\
Residential environment & Training & 387 & 46 \\
& Testing & 467 & 85 \\
Child interacting with instructor & School & 412 & 97.73 \\
Using apps & College & 97 & 21.96 \\
\hline
\end{tabular}

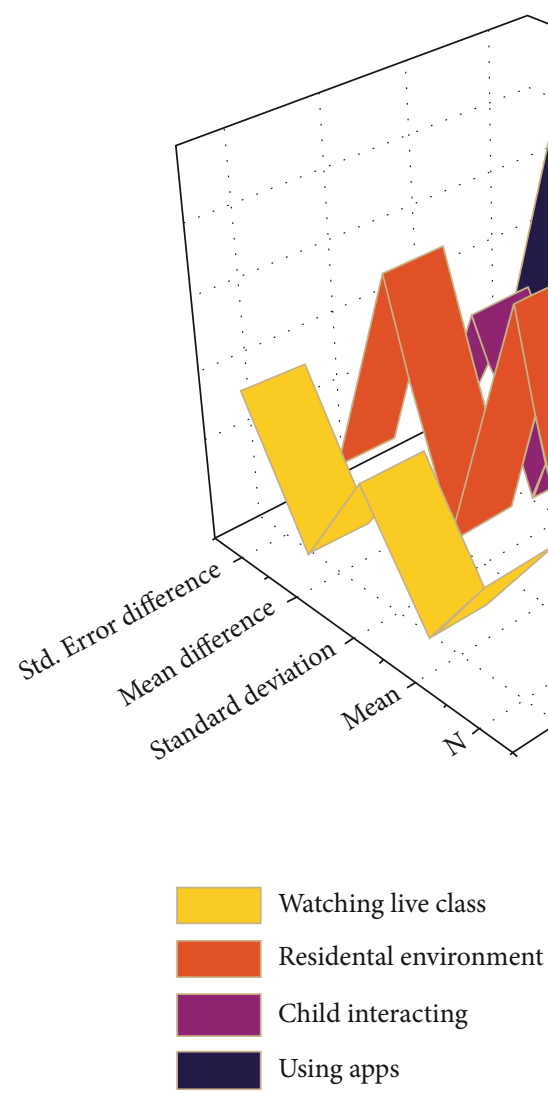

FIgURE 5: Impartial measurement mobile learning on the optimization higher education; $N$ represents for number of students.

TABLE 4: Result impartial measurement mobile learning on the optimization $p$.

\begin{tabular}{llccccc}
\hline Parameters & Group & $N$ & Mean & S. D. & Mean difference & Std. error difference \\
\hline Watching live class & School/college & 28.8 & 3.19 & 1.25 & -0.45 & 0.16 \\
Residential environment & School/college & 32.5 & 2.15 & 1.45 & -0.89 & 0.16 \\
Child interacting with instructor & School/college & 37.2 & 2.67 & 2.76 & 0.05 & 0.35 \\
Using apps & School/college & 38.9 & 2.91 & 2.12 & -0.23 & 0.35 \\
\hline
\end{tabular}

evaluation and selection but will also turn big data size evaluation and selection of all school information into a tool for user will be notified. Educational leaders and teachers can recognize inadequacies in students' knowledge as a result of large data analysis or rather choice evaluation of the student, and personalized teaching is decided to carry out for schools depending on the vulnerabilities of students' understanding, which further improves the level of education.
Figure 4 clearly demonstrates how the precision of higher education measurement and selection under data mining evolves over time. The greater the time span, the greater the accuracy of higher learning measurement and selection, which has been ensured to the maximum extent possible. It also verifies the overall effectiveness of the information higher education analysis and decision-making method (Table 3). 
The study's population was selected in a nonprobabilistic manner. It consisted of students from its most critical extracurricular activities - the dataset comprised 576 extracurricular activity participants and 509 children interacting with instructor participants. Female education programmers comprise the majority of respondents (Table 3). However, 345 (85.65\%) students are male, and $573(81.53 \%)$ are female, $412(97.73 \%)$ were from a child interacting with the school, and $97(21.96 \%)$ are from a child interacting with the college.

In spite of the challenges they encountered, students believe that traditional face-to-face method is the best way to carry out the entire teaching and learning process, and also that the platform should be used as a supplement to facilitate the educational process (Figure 5). Thus, $68.52 \%$ of students prefer expressions teaching/learning, $52.37 \%$ prefer a mix of traditional and digital classes, and 18.63 percent prefer online-based educational (Table 4).

When applied to a learning platform, the higher education analysis and stance method obtained through data mining can indeed effectively carry out intelligent simultaneous evaluation and selection and also create large data evaluation but also selection of all educational methods into the method for user notification. Big data measurement and stance analysis of a classroom can identify the weak points of students' knowledge, and highly personalized teaching is chosen to carry out for students based on the weak points of students' understanding, which further enhances the teaching quality. From this, we can observe ANN is the best one to analyze mobile learning on the optimization of teaching mode in higher education. An artificial neural network would be an effort to simulate the network of neurons that encompass the brain activity for the computer to learn and make judgments similar to a human's circumstance. ANNs are generated by processing frequent machines to act like an interrelated central nervous system.

\section{Conclusion}

Mobile learning is employed to optimize the teaching mode of higher education, which provides a new direction for the reform of higher education under the rapid development of the Internet. The study of the current development of higher education reveals that the dependence of contemporary college students on mobile devices has gradually increased with the continuous progress of science as well as technology, and the TTM cannot meet the learning needs of college students. This study employed artificial neural network algorithm for enhancing the effect of mobile learning in higher education. Mobile learning is based on mobile device terminal, and new teaching methods are adopted to provide new learning conditions for college students. The reason may be that the way of mobile learning is relatively novel, which can effectively promote students' learning interest. Moreover, mobile terminals can help students improve classroom participation and interaction with teachers and enjoy fun while learning knowledge in the classroom, which is an effective means to improve their learning enthusiasm. Hence, compared with the TTM, mobile learning can improve students' learning enthusiasm.

\section{Data Availability}

The data used to support the findings of this study are available from the corresponding author upon request.

\section{Conflicts of Interest}

The author declares no conflicts of interest.

\section{References}

[1] M. A. Al-Mashhadani and M. F. Al-Rawe, "The future role of mobile learning and smartphones applications in the Iraqi private universities," Smart Learning Environments, vol. 5, no. 1, pp. 1-11, 2018.

[2] H. F. El-Sofany and N. El-Haggar, "The effectiveness of using mobile learning techniques to improve learning outcomes in higher education," International Journal of Interactive Mobile Technologies (iJIM), vol. 14, no. 8, p. 4, 2020.

[3] D. Darmaji, D. Kurniawan, A. Astalini, A. Lumbantoruan, and S. Samosir, Mobile Learning in Higher Education for the Industrial Revolution 4.0: Perception and Response of Physics Practicum, International Association of Online Engineering, 2019.

[4] C. Klein and R. M. Hess, "Using Learning Analytics to Improve Student Learning Outcomes Assessment," in Learning Analytics in Higher Education, pp. 140-159, Routledge, 2018.

[5] R. Kaliisa and M. Picard, "A systematic review on mobile learning in higher education: the African perspective," TOJET: The Turkish Online Journal of Educational Technology, vol. 16, no. $1,2017$.

[6] P. Prasertsilp, "Mobile learning: designing a socio-technical model to empower learning in higher education," LUX: A Journal of Transdisciplinary Writing and Research from Claremont Graduate University, vol. 2, no. 1, pp. 1-12, 2013.

[7] Y. Gupta, F. M. Khan, and S. Agarwal, "Exploring factors influencing mobile learning in higher education - a systematic review," International Journal of Interactive Mobile Technologies (iJIM), vol. 15, no. 12, p. 140, 2021.

[8] J. M. Romero-Rodríguez, I. Aznar-Díaz, F. J. Hinojo-Lucena, and M. P. Cáceres-Reche, "Models of good teaching practices for mobile learning in higher education," Palgrave Communications, vol. 6, no. 1, pp. 1-7, 2020.

[9] J. M. Romero-Rodríguez, I. Aznar-Díaz, F. J. Hinojo-Lucena, and G. Gómez-García, "Mobile learning in higher education: structural equation model for good teaching practices," Ieee Access, vol. 8, pp. 91761-91769, 2020.

[10] J. J. Willemse, K. Jooste, and V. Bozalek, "Experiences of undergraduate nursing students on an authentic mobile learning enactment at a higher education institution in South Africa," Nurse Education Today, vol. 74, pp. 69-75, 2019.

[11] H. C. H. Wong, "A study of mobile learning for higher education students in Guangzhou," Springerplus, vol. 3, Supplement 1, pp. 1-2, 2014.

[12] N. Uosaki, H. Ogata, K. Mouri, and M. Choyekh, "Implementing Sustainable Mobile Learning Initiatives for Ubiquitous Learning Log System Called SCROLL," in Education in the Asia-Pacific Region: Issues, Concerns and Prospects, pp. 89-114, Springer, Singapore, 2017.

[13] R. Zeng and E. Luyegu, Mobile Learning in Higher Education. In Informed Design of Educational Technologies in Higher Education: Enhanced Learning and Teaching, IGI Global, 2012. 
[14] H. Shuang and M. David, "From point to surface': the role of policy experimentation in Chinese higher education reforms," British Journal of Educational Studies, vol. 69, no. 2, pp. 217236, 2021

[15] M. Antoniadou and M. Kathleen, "Holding true or caving in? Academics' values, emotions, and behaviors in response to higher education reforms," Higher Education Policy, vol. 4, no. 72, p. 35, 2021.

[16] S. Joshua, "Wasted talents? China's higher education reforms experienced through its visiting scholars abroad," Journal of Contemporary China, vol. 36, no. 42, p. 74, 2021.

[17] L. Zhao, "The analysis of higher education reform strategy based on the new situation of network," Education Reform and Development, vol. 2, no. 2, p. 31, 2020. 